\title{
Proprietary arabinogalactan extract increases antibody response to the pneumonia vaccine: a randomized, double-blind, placebo-controlled, pilot study in healthy volunteers
}

Jay K Udani ${ }^{1,2^{*}}$, Betsy B Singh ${ }^{1}$, Marilyn L Barrett ${ }^{3}$, Vijay J Singh ${ }^{1}$

\begin{abstract}
Background: Arabinogalactan from Larch tree (Larix spp.) bark has previously demonstrated immunostimulatory activity. The purpose of this study was to test the hypothesis that ingestion of a proprietary arabinogalactan extract, ResistAid ${ }^{T M}$, would selectively enhance the antibody response to the pneumococcal (pneumonia) vaccine in healthy adults.

Methods: This randomized, double-blind, placebo-controlled, parallel group pilot study included 45 healthy adults who had not previously been vaccinated against Streptococcus pneumoniae. The volunteers began taking the study product or placebo (daily dosage $4.5 \mathrm{~g}$ ) at the screening visit (V1-Day 0) and continued over the entire 72 day study period. After 30 days the subjects received the 23-valent pneumococcal vaccine (V2). They were monitored the following day (V3-Day 31), as well as 21 days (V4-Day 51) and 42 days (V5-Day 72) after vaccination. Responses by the adaptive immune system (antigen specific) were measured via pneumococcal IgG antibodies (subtypes 4 , $6 \mathrm{~B}, 9 \mathrm{~V}, 14,18 \mathrm{C}, 19 \mathrm{~F}$, and 23F) and salivary lgA levels. Responses by the innate immune system (non-specific) were measured via white blood cell counts, inflammatory cytokines and the complement system.

Results: Vaccination significantly increased pneumococcal lgG levels as expected. The arabinogalactan group demonstrated a statistically significant greater lgG antibody response than the placebo group in two antibodies subtypes ( $18 \mathrm{C}$ and 23F) at both Day $51(p=0.006$ and $p=0.002)$ and at Day $72(p=0.008$ and $p=0.041)$. These same subtypes (18C and 23F) also demonstrated change scores from baseline which were significant, in favor of the arabinogalactan group, at Day $51(p=0.033$ and 0.001$)$ and at Day $72(p=0.012$ and $p=0.003)$. Change scores from baseline and mean values were greater in the arabinogalactan group than placebo for most time points in antibody subtypes $4,6 \mathrm{~B}, 9 \mathrm{~V}$, and 19F, but these differences did not reach statistical significance. There was no effect from the vaccine or arabinogalactan on salivary lgA, white blood cell count, inflammatory cytokines or complement.
\end{abstract}

Conclusions: The proprietary arabinogalactan extract (ResistAid ${ }^{T M}$ ), tested in this randomized, double-blind, placebo-controlled, parallel-group pilot study, increased the antibody response of healthy volunteers to the 23-valent pneumococcal vaccine compared to placebo.

Trial Registration: ISRCTN98817459

* Correspondence: jay.udani@medicusresearch.com

${ }^{1}$ Medicus Research LLC, Northridge, CA 91325, USA

Full list of author information is available at the end of the article 


\section{Background}

The immune system is a highly complex orchestration of cells, organs, tissues and active molecules which interact in an elaborate and dynamic network to protect the body from infection. The immune system can be divided into two categories: the innate immune system and the adaptive immune system. Innate immunity is an immediate but non-specific response. Adaptive or acquired immunity involves a specific reaction to a pathogen which the immune system recognizes from a previous encounter. The process of acquired immunity is the basis for vaccination[1]. Recent research has focused on the role of nutrition (foods and specific components of foods) in the responsiveness of the immune system to challenges. Vaccine-specific serum antibody production has been suggested as a highly suitable model to evaluate dietary intervention on the resistance to infection or to other immune system-related diseases[2].

The pneumococcal vaccine can reduce the incidence and/or severity of infections caused by Streptococcus pneumoniae: namely, pneumonia, otitis media, sinusitis and meningitis. The 23-valent vaccine contains 23 pneumococcal polysaccharide antigens (serotypes $1,2,3,4,5$, $6 \mathrm{~B}, 7 \mathrm{~F}, 8,9 \mathrm{~N}, 9 \mathrm{~V}, 10 \mathrm{~A}, 11 \mathrm{~A}, 12 \mathrm{~F}, 14,15 \mathrm{~B}, 17 \mathrm{~F}, 18 \mathrm{C}$, 19A, 19F, 20, 22F, 23F and 33F[3]. Although there are at least 90 distinct serotypes, these 23 serotypes accounted for $85 \%$ to $90 \%$ of invasive pneumococcal infections in the US[3]. The 23-valent vaccine produces a humoral (antibody-mediated) response: inducing the production of antibody from B-lymphocytes in the absence of help from T-lymphocytes. The type and concentration of antibody produced is dependent on the site of exposure. Systemic administration results primarily in the generating of circulating immunoglobulin(Ig)G whereas mucosal antigenic challenge results in a more vigorous IgA response[1]. In contrast to the 23-valent pneumococcal vaccine, a 7 -valent vaccine conjugated to a nontoxic diphtheria protein (used for children younger than 5 years) will induce a T-cell response[3].

Studies on improvement of the response to the pneumococcal vaccine by adults include revaccination, the addition on conjugates to the vaccine and alternative antigenic substances[4]. In addition, nutritional products have been tested on their effect on the response to vaccination. Supplementation with $200 \mathrm{mg} /$ day vitamin E for 4 months to subjects at least 65 years of age caused a suggestive, but insignificant, increase in antibody response to the pneumococcal vaccine[5]. Another study evaluated the effects of prebiotic fructo-oligosaccharides (70\% raftilose and 30\% raftiline) derived from inulin on the response by an elderly population (70 years old and above). In this study the response to vaccination with the influenza $B$ and pneumococcal vaccines was not significantly increased[6].

Arabinogalactans are high molecular weight, highly branched, water-soluble polysaccharides, which contain units of D-galactose and L-arabinose[7]. Arabinogalactans have previously demonstrated immunostimulatory activity $[8,9]$. They are present in several immune-enhancing herbs, including Echinacea purpurea, Baptisia tinctoria, Thuja occidentalis, Angelica acutiloba, and Curucuma longa and the medicinal mushroom Ganoderma lucidum.[10-12]. Arabinogalactans from Larch (Larix spp.) have been shown to stimulate natural killer cell cytotoxicity in vitro through the generation of interferon gamma and inhibit the metastasis of tumor cells to the liver in a rodent model[7,13,14]. A dog study demonstrated increases in white blood cell counts (due to increases in neutrophils and eosinophils), and no effect on serum IgG, IgM or IgA following oral adminstration indoses of $0.55 \mathrm{~g} /$ day or $1.65 \mathrm{~g} /$ day for 10 days [15]. A randomized, double-blind, placebo-controlled study evaluated the immunomodulating effects of a preparation of proprietary larch arabinogalactan ( $1.5 \mathrm{~g} /$ day) alone, and in combination with various preparations of Echinacea: an extract of Echinacea purpurea whole herb containing $4 \%$ phenolic compound ( $1.5 \mathrm{~g} /$ day), a preparation of $E$. purpurea whole herb and a preparation E. angustifolia root (36 to $680 \mathrm{mg} /$ day)[16]. The study included 48 adult women who were divided into six groups of eight women. After 4 weeks of treatment, complement properdin increased by $18 \%$ in the group that received all four preparations and by $21 \%$ in the group given preparations of both species of Echinacea.

The current human clinical pilot study was designed to test the hypothesis that the ingestion of Resistaid ${ }^{\mathrm{m}}$, a proprietary arabinogalactan extracted from Larch (Larix laricina), would selectively enhance the antibody response by adults to the 23 -valent pneumococcal vaccine. Indications that the product would have immunostimulatory activity came from previous studies conducted with this proprietary product $[15,16]$. As there was no prior human data regarding the ability of this proprietary arabinogalactan extract to impact the immune response to the pneumococcal vaccine, a power calculation could not be performed. The sample size was set at a level consistent with prior human studies involving arabinogalactan and the immune system [16-18].

\section{Methods \\ Investigational products}

The proprietary arabinogalactan product ResistAid ${ }^{\mathrm{Tw}}$, supplied by Lonza Ltd, Switzerland, contains 
arabinogalactan extracted from Larch (Larix laricina). Arabinogalactan is a highly branched polysaccharide that is composed of galactose units and arabinose units in the approximate ratio of 6:1[7]. ResistAid ${ }^{\text {tw }}$ is a fine, dry, light brown powder with a neutral taste that dissolves quickly in water or juice. ResistAid ${ }^{\text {ta }}$ is produced via a water extraction patented process (US 5756098; EP 86608), in accordance with Hazard Analysis and Critical Control Points (HACCP) standards and in compliance with the monograph in the Food Chemicals Codex. The Good Manufacturing Practices (GMP's) used during manufacturing are audited by the American Institute of Baking. The Larch arabinogalactan used in the ResistAid ${ }^{\text {tw }}$ product has been designated as Generally Recognized as Safe (GRAS) with the US FDA (GRAS Notice Nos. GRN000047 and GRN000084).

The placebo was maltodextrin (Maltrin M100, Grain Processing Corp., USA). The test product and the placebo were administered by mixing the powders into a beverage of the subject's choice. The subjects were advised to take their dosage ( $4.5 \mathrm{~g})$ once a day in the morning with breakfast. They began taking their assigned powder on Day 1 and continued over the entire 72 day study period.

\section{Subjects}

Subjects between the ages of 18 and 65 were recruited for the study in the usual manner (subject database and community advertisements). Subjects were phonescreened prior to scheduling a screening visit.

Subjects were included if they were 18-65 years of age, had a Body Mass Index (BMI) $\geq 18 \mathrm{~kg} / \mathrm{m}^{2}$ and $\leq 30 \mathrm{~kg} /$ $\mathrm{m}^{2}$ at screening, agreed to all study visits and visit procedures, agreed to use approved forms of birth control, and agreed not to initiate/change any exercise or diet programs during the study. Subjects were excluded if they had previously had the pneumococcal vaccine, had any major systemic, inflammatory or chronic disease, had any active infection or infection in the past month requiring antibiotics or anti-viral medication, used immunosuppressive drugs in the prior 5 years, were known to have alcohol or drug abuse, were pregnant or lactating or had any medical condition which in the opinion of the investigator might interfere with the subject's participation in the trial.

\section{Study Design}

The study was a randomized, double-blind, placebo-controlled, parallel group trial with an active investigational period of 72 days. The objective was to assess the immunomodulatory effect of the arabinogalactan product on selective markers of immune function following antigenic challenge by the pneumococcal vaccine. The primary endpoints were 7 different pneumococcal IgG antibodies. The secondary objective was to determine whether the arabinogalactan product would stimulate other arms of the immune system to which there was no direct antigenic stimulus. Secondary endpoints included salivary IgA, white blood cell counts, complement (C3 and $\mathrm{C} 4)$ and inflammatory cytokine levels. The study was conducted at the Staywell Research clinical research site located in Northridge, CA and was designed and managed by the Medicus Research Contract Research Organization (CRO) also in Northridge, CA. IRB approval was obtained prior to the initiation of any study activities (Copernicus Group IRB, Cary, NC).

Subjects meeting all of the inclusion criteria and none of the exclusion criteria for this study were randomly assigned to receive either the arabinogalactan or placebo. Double-blinding was ensured by the use of identical opaque sachets, outer packaging, labelling and color for both investigational products (arabinogalactan and placebo). Unblinding of the entire research team, including data analysis team did not occur until after the analysis was completed; subjects were blinded throughout the trial.

The study began in August 2008 (first subject in) and lasted until December 2008 (last subject completed). The subjects in the study came to the research clinic for a total of 5 visits (V1-V5) over 72 days. Subjects took the first dose of assigned study product at the screening visit (V1-Day 0) and continued to take them over the entire study. They received the 23-valent pneumococcal vaccine (Pneumovax ${ }^{\circ}$ 23, Merck and Co., Inc., USA) at the vaccine visit which took place 30 days after they began taking the product or placebo (V2-Day 30). They came in for safety monitoring the day immediately following the vaccine (V3-Day 31) to observe the reaction at the vaccine administration site. Then subjects returned 21 days after vaccine (V4-Day 51) and finally 42 days after vaccine administration (V5-Day 72). On study visits, blood, urine and saliva were collected and subjects were queried regarding any change in health status. Additionally, they were assessed for compliance by interview, diary, and through the return of unused study product sachets.

The most potentially immunogenic pneumococcal antibodies $(\mathrm{Ab})$ were determined in consultation with the UCLA Vaccine Center (Torrance, CA, USA) as the antibodies most likely to respond to vaccination with the 23-valent pneumococcal vaccine. These antibodies included 4, 6B, 9V, 14, 18C, 19F, and 23F. Salivary IgA was measured to monitor for non-specific effects on the adaptive immune system using immuno-array assays with a minimum sensitivity of $1.0 \mu \mathrm{g} / \mathrm{ml}$. Other markers of immune function were chosen to represent the innate arm of the immune system including white blood cell counts (totals and subtypes), inflammatory cytokines, 
and complement ( $\mathrm{C} 3$ and $\mathrm{C} 4$ ) determined using immuno-turbidimetric methodology. Analysis of inflammatory cytokine levels were performed using sandwich immunoassay (Affymetrix, San Diego, CA, USA). Safety monitoring included: body temperature, blood pressure, heart rate, physical exam, urinalysis, complete blood counts $(\mathrm{CBC})$ and a comprehensive metabolic panel (CMP) including kidney and liver function tests.

\section{Analyses}

Excel 2003 (Microsoft Corp, Redmond WA, USA), was used for data entry, validation, restructuring, calculating changes in variables over time, reorganizing and reformatting results, and preparing graphs. Statistical analyses were performed using SPSS Base System ver. 17 (SPSS Inc., Chicago IL, USA).

Data was analyzed using paired sample t-tests for within subject means comparisons, independent sample t-tests for between group comparisons (placebo vs. the active groups individually). Difference scores for both within and between group comparisons (placebo vs. the active groups individually) were analyzed using appropriate t-tests. Analysis was completed before the blinding code was broken.

\section{Results}

\section{Subjects}

Sixty five (65) subjects were screened in person at the research clinic and 53 qualified for randomization at the screening visit (V1). Of the 53, 8 did not return for V2 and therefore a total of 45 subjects were included in the intent-to-treat analysis. The subject baseline characteristics are given in Table 1.

\section{Pneumococcal IgG antibodies}

Pneumococcal IgG antibody subtypes 4, 6B, 9V, 14, 18C, 19F, and 23F were measured on Days 0 (V1), 51 (V4), and 72 (V5). There were no significant differences between the groups at baseline (Day 0 ).

Pneumococcal IgG levels increased from baseline in response to the vaccine as expected. Supplementation with the arabinogalactan product caused a significantly greater increase from baseline in pneumococcal IgG antibody subtypes $18 \mathrm{C}$ and $23 \mathrm{~F}$ at both 51 and 72 days (Table 2). Mean values between groups were also significantly greater in the arabinogalactan group for both days 51 and 72 for these two subtypes (Table 3).

Table 1 Subject Demographics

\begin{tabular}{ccc}
\hline & ResistAid $^{\left({ }^{(\mathbf{m})}\right.}$ & Placebo \\
\hline $\mathbf{N}$ & 21 & 24 \\
\hline Male & $9(42.9 \%)$ & $16(66.7 \%)$ \\
\hline Female & $12(57.1 \%)$ & $8(33.3 \%)$ \\
\hline Age (range) & $33.52(19-62)$ & $38.25(20-64)$ \\
\hline
\end{tabular}

Change scores from baseline and mean values were greater in the arabinogalactan group than placebo for most time points in $\mathrm{Ab}$ subtypes $4,6 \mathrm{~B}, 9 \mathrm{~V}$, and $19 \mathrm{~F}$, but these differences did not reach statistical significance.

\section{Salivary $\lg A$}

Salivary IgA levels in the placebo group were $146 \pm$ $109 \mathrm{mg} / \mathrm{dl}$ at baseline (Day 0). There were no significant changes from Day 0 to Days 51 or Day 0 to Day 72 in either group. There were also no significant differences in the mean values between groups.

\section{White blood cells}

The mean total white blood cell count was $6.50 \pm 1.46 \times$ $1000 / \mu \mathrm{l}$ in the placebo group at baseline (Day 0). Comparisons between the arabinogalactan and placebo groups on Days $0,30,31,51$ or 72 found no significant differences in total white blood cell counts. The change from baseline Day 0 to Day 72 was significantly greater in the arabinogalactan group than the placebo group $(0.38 \pm 0.79$ compared to $0.15 \pm 1.33 ; p=0.045)$.

Differential analysis of white blood cells determined that the levels at baseline were as follows: neutrophils $63.1 \pm 5.3$, lymphocytes $28.4 \pm 6.0$, monocytes $6.9 \pm 1.9$, eosinophils $1.6 \pm 1.5$ and basophils $0.33 \pm 0.56$ (measured as a percent of total white blood cells). There were no significant differences in lymphocyte, neutrophil, monocyte, or basophil counts when comparing mean values between groups at any time point. When comparing change from baseline at each time point, there were no differences between groups for lymphocytes, neutrophils, or monocytes. Change from baseline comparisons for basophils revealed a statistically significant, but clinically insignificant increase in numbers in the placebo group compared to the arabinogalactan group when comparisons were made between Day 0 and Day $72(0.21 \pm 0.72$ placebo compared to $0.09 \pm 0.54$ arabinogalactan; $\mathrm{p}=0.042$ ).

Eosinophil counts were different between groups on Day $30(2.81 \pm 2.04$ vs $1.46 \pm 0.98 ; \mathrm{p}=0.006)$ and on Day $51(3.24 \pm 2.12$ vs $1.83 \pm 1.55 ; \mathrm{p}=0.014)$ with higher numbers in the arabinogalactan group. There was a larger increase in cell number (change) from baseline to Day $31(0.14 \pm 1.39$ vs $0.83 \pm 0.72 ; \mathrm{p}=0.035)$ and from baseline to Day $51(0.48 \pm 1.69$ vs $0.20 \pm 0.66$; $\mathrm{p}=0.006)$ in the arabinogalactan group.

\section{Complement}

The levels of complement C3 and C4 at Day 0 were $125 \pm 23$ and $28 \pm 10 \mathrm{mg} / \mathrm{dl}$, respectively. Comparisons of means and changes from baseline for complement $(\mathrm{C} 3, \mathrm{C} 4)$ levels between the arabinogalactan and placebo groups were not significantly different. 
Table 2 Effects of the 23-valent vaccine on Pneumococcal IgG antibodies

\begin{tabular}{lccccc}
\hline Antibody subtype & $\begin{array}{c}\text { Day 0 } \\
\text { Mean } \pm \text { SD }\end{array}$ & $\begin{array}{c}\text { Day 51 } \\
\text { Mean } \pm \text { SD }\end{array}$ & $\begin{array}{c}\text { Day 72 } \\
\text { Mean } \pm \text { SD }\end{array}$ & $\begin{array}{c}\text { Change } \\
\text { Days 0-51 }\end{array}$ & $\begin{array}{c}\text { Change } \\
\text { Days 0-72 }\end{array}$ \\
\hline Type 4 & $0.45 \pm 0.64$ & $2.21 \pm 3.15$ & $5.84 \pm 7.35$ & 0.023 & 0.042 \\
\hline Type 6B & $0.95 \pm 1.51$ & $5.18 \pm 6.64$ & $5.19 \pm 7.06$ & 0.001 & 0.020 \\
\hline Type 9V & $1.32 \pm 4.10$ & $6.07 \pm 7.34$ & $5.08 \pm 5.25$ & 0.129 & 0.095 \\
\hline Type 14 & $1.79 \pm 2.56$ & $9.91 \pm 8.54$ & $8.86 \pm 8.59$ & 0.000 & 0.006 \\
\hline Type 18C & $0.72 \pm 1.35$ & $5.06 \pm 5.80$ & $4.93 \pm 5.26$ & 0.018 & 0.006 \\
\hline Type 19F & $1.10 \pm 2.94$ & $7.02 \pm 7.28$ & $6.65 \pm 7.26$ & 0.011 & 0.015 \\
\hline Type 23F & $1.08 \pm 1.87$ & $4.32 \pm 4.62$ & $4.55 \pm 5.23$ & 0.017 & 0.006 \\
\hline
\end{tabular}

Increases in levels of antibody subtype as observed in the placebo group $(n=24)$ following inoculation with the 23 -valent pneumococcal vaccine which took place on Day 30. Data are means $(\mu \mathrm{g} / \mathrm{dl}) \pm$ standard deviations on Days 0,51 and 72 . P-values for the changes between baseline and days 51 and 72 are in the right hand columns.

\section{Cytokines}

Comparison of cytokine levels between groups found no significant differences in means for epithelial neutrophilactivating peptide (ENA)-78, eotaxin, granulocyte monocyte colony stimulating factor (GM-CSF), interferongamma (IFNg), interleukin (IL)-10, IL-12P40, IL-1RA, IL-2, IL-4, IL-5, IL-6, IL-8, monocyte chemotactic protein (MCP)-1, MCP-3, platelet-derived growth factor (PDGF)-BB or tumor necrosis factor (TNF)-alpha. When comparing the cytokine change from baseline values between groups, only the IL-6 change from Day 30 to Day 31 showed an increase in the arabinogalactan group compared to the placebo group. The change in the arabinoglactan group was from a mean of $17.8 \pm 7.7$ to $19.8 \pm 7.7 \mathrm{pg} / \mathrm{ml}(+1.9)$, compared to a change from $50.1 \pm 113.8$ to $48.7 \pm 112.5 \mathrm{pg} / \mathrm{ml}$ for the placebo group $(-2.4)(\mathrm{p}=0.046)$. This was most likely in response to the vaccine which was administered on Day 30.

\section{Safety}

No serious adverse events were reported during this study. There were nine mild adverse events in the placebo group (erythema at injection site (1), sore throat
(2), nasal congestion (3), headache (2), and abdominal pain (1). There were no adverse events in the active group. All adverse events were followed by the medical staff at the research clinic.

\section{Discussion}

The results of this pilot study suggest that the arabinogalactan preparation had a selective immunostimulating effect on acquired or adaptive immunity, as shown in the increase in antibodies without any clinically significant effects on total white blood cells, cytokines or complement. Thus it is possible that rather than acting as a general immunostimulant, arabinogalactan acted in a specific manner. The caveat to this statement is that these results are preliminary and there are confounding variables to consider.

Variables that affect the immune response to vaccines include age, gender, race and genetic characteristics[19]. One of the goals of this pilot study was to determine the effect of the intervention on a relatively broad population. As such, the study population included males and females from age 18 to 65 . The randomization scheme was sequential and therefore the gender of subjects was not matched in advance. As gender and age

Table 3 Pneumococcal IgG types $18 \mathrm{C}$ and 23F - Comparisons between ResistAid ${ }^{(\mathrm{TM})}$ and Placebo Groups

\begin{tabular}{|c|c|c|c|c|c|}
\hline & $\begin{array}{c}\text { Day } 0 \\
\text { Mean } \pm \text { SD }\end{array}$ & $\begin{array}{c}\text { Day } 51 \\
\text { Mean } \pm \text { SD }\end{array}$ & $\begin{array}{c}\text { Day } 72 \\
\text { Mean } \pm \text { SD }\end{array}$ & $\begin{array}{c}\text { Change } \\
\text { Day 0-51 }\end{array}$ & $\begin{array}{c}\text { Change } \\
\text { Day 0-72 }\end{array}$ \\
\hline \multicolumn{6}{|l|}{ Type $18 \mathrm{C}$} \\
\hline ResistAid $^{T M}(n=21)$ & $1.49 \pm 3.00$ & $9.57 \pm 7.96$ & $9.10 \pm 7.53$ & $8.08 \pm 7.12$ & $7.61 \pm 6.81$ \\
\hline Placebo $(n=24)$ & $0.72 \pm 1.35$ & $5.06 \pm 5.80$ & $4.93 \pm 5.26$ & $4.34 \pm 5.10$ & $4.22 \pm 4.69$ \\
\hline Comparison ( $p$-value) & 0.061 & 0.006 & 0.008 & 0.033 & 0.012 \\
\hline \multicolumn{6}{|l|}{ Type 23F } \\
\hline ResistAid $^{T M}(n=21)$ & $0.74 \pm 0.93$ & $7.07 \pm 7.41$ & $7.02 \pm 7.31$ & $6.33 \pm 7.36$ & $6.28 \pm 7.17$ \\
\hline Placebo $(n=24)$ & $1.08 \pm 1.87$ & $4.32 \pm 4.62$ & $4.55 \pm 5.23$ & $3.24 \pm 4.28$ & $3.46 \pm 4.24$ \\
\hline Comparison ( $p$-value) & 0.059 & 0.002 & 0.041 & 0.001 & 0.003 \\
\hline
\end{tabular}

Levels of antibody subtypes $18 \mathrm{C}$ and $23 \mathrm{~F}$ in the ResistAid ${ }^{\mathrm{TM}}$ and placebo groups are given as means ( $\left.\mu \mathrm{g} / \mathrm{dl}\right) \pm \mathrm{standard}$ deviations on Days 0,51 and 72 and changes from Day 0 . Inoculation with the 23-valent pneumococcal vaccine took place on Day 30. P-values are comparisons between groups and comparisons of changes from Day 0 in the two groups. 
differences may affect immunity these potentially confounding variable should be looked at in future studies. This study was an exploratory investigation into the effects of arabinogalactan with the goal of determining whether further studies are warranted. The result is that further studies with larger populations are indicated to clarify and potentially expand upon the effects of arabinogalactan on antibody production.

The suggestion that arabinogalactan might have a selective effect on the immune system is preliminary but promising. The immune system entails a complex matrix of responses to protect the body from pathogens and toxins. The innate immune system involves the rapid recruitment and upregulation of neutrophils, monocytes, macrophages, complement factors, cytokines and antimicrobial peptides to the site of infection. The innate response is the first line of host defense and the adaptive response follows a few days later. While the innate and adaptogenic arms of the immune system are often described as separate, they often act together in a synergistic manner[20]. In addition to antibodies, the variables tested in this study included salivary IgA, white blood cell counts (lymphocytes, neutrophils, monocytes, basophils and eosinophils), complement C-3 and C-4 as well as numerous cytokines. Additionally, suggestions of changes were observed in IL-6 levels and in eosinophil counts. IL-6 has immunostimulatory properties and eosinophils play a role in allergic responses. The clinical significance of these findings is unknown at this time. Additional measurements for future studies could include a breakdown of lymphocytes into subtypes, measuring natural killer (NK) lymphocytes and NK-T cells. NK cells are a heterogeneous population of innate $\mathrm{T}$ cells that have attracted interest because of their potential to regulate immune responses to a variety of pathogens and NK-T cells function as a bridge between innate and adaptive immunity[20].

Arabinogalactan was given for 30 days prior to vaccination and administration was continued throughout the study. The 30 days time period was chosen because a previous clinical trial studying the effect of arabinogalactan and echinacea preparations on the immune system observed a positive effect following treatment for this period of time[16].

This is the first human study to demonstrate an effect by Larch arabinogalactan on immunoglobulin levels. No effect on IgG antibody levels was observed in another study wherein the subjects were administered $1.5 \mathrm{~g}$ larch arabinogalactan per day for four weeks[16]. This study utilized a larger dose (4.5 g per day), longer administration time (10 weeks) and the vaccine as a standardized antigenic challenge all of which appear to have been useful in delineating a difference between the proprietary arabinogalactan extract and placebo.

\section{Conclusions}

The proprietary arabinogalactan extract (ResistAid ${ }^{\mathrm{TM}}$ ) tested in this randomized, double-blind, placebo-controlled, parallel-group study, increased the antibody response of healthy volunteers to the 23 -valent pneumococcal vaccine compared to placebo. The proprietary arabinogalactan product was administered safely in this study in a dose of $4.5 \mathrm{~g}$ per day for approximately 10 weeks. This was a pilot study that demonstrated promising effects and further studies with larger populations are indicated which may demonstrate additional effects of arabinogalactan on the immune system.

\section{Acknowledgements}

Medicus Research would like to thank Lonza Inc., of Allendale, NJ, for supplying the investigational products and providing financial support for all aspects of this clinical study.

\section{Author details}

${ }^{1}$ Medicus Research LLC, Northridge, CA 91325, USA. ²UCLA School of Medicine, Department of Medicine, Los Angeles, CA 90024, USA.

${ }^{3}$ Pharmacognosy Consulting, Mill Valley, CA 94941, USA.

\section{Authors' contributions}

JKU conceptualized the study and was the Principal Investigator. BBS also participated in the design of the study. BBS and VJS performed the analysis. JKU, BBS and MLB contributed to writing the manuscript. All authors have read and approved the final manuscript.

\section{Competing interests}

The authors declare that they have no competing interests.

Received: 1 September 2009 Accepted: 26 August 2010

Published: 26 August 2010

\section{References}

1. Twigg HL III: Humoral immune defense (antibodies): recent advances. Proc Am Thorac Soc 2005, 2:417-421.

2. Albers R, Antoine JM, Bourdet-Sicard R, Calder PC, Gleeson M, Lesourd B, Samartín S, Sanderson IR, Van Loo J, Vas Dias FW, Watzl B: Markers to measure immunomodulation in human nutrition intervention studies. $\mathrm{Br}$ J Nutr 2005, 94:452-481.

3. Targonski PV, Poland GA: Pneumococcal vaccination in adults: recommendations, trends, and prospects. Cleve Clin J Med 2007, 74:401-10, 413.

4. Artz AS, Ershler WB, Longo DL: Pneumococcal vaccination and revaccination of older adults. Clin Microbiol Rev 2003, 16:308-318.

5. Meydani SN, Meydani M, Blumberg JB, Leka LS, Siber G, Loszewski R, Thompson C, Pedrosa MC, Diamond RD, Stollar BD: Vitamin E supplementation and in vivo immune response in healthy elderly subjects. A randomized controlled trial. JAMA 1997, 277:1380-1386.

6. Bunout D, Hirsch S, Pia dIM, Munoz C, Haschke F, Steenhout P, Klassen P, Barrera G, Gattas $V$, Petermann M: Effects of prebiotics on the immune response to vaccination in the elderly. JPEN J Parenter Enteral Nutr 2002, 26:372-376.

7. Kelly GS: Larch arabinogalactan: clinical relevance of a novel immuneenhancing polysaccharide. Altern Med Rev 1999, 4:96-103.

8. Beuth J, Ko HL, Oette K, Pulverer G, Roszkowski K, Uhlenbruck G: Inhibition of liver metastasis in mice by blocking hepatocyte lectins with arabinogalactan infusions and D-galactose. J Cancer Res Clin Oncol 1987, 113:51-55.

9. Beuth J, Ko HL, Schirrmacher V, Uhlenbruck G, Pulverer G: Inhibition of liver tumor cell colonization in two animal tumor models by lectin blocking with D-galactose or arabinogalactan. Clin Exp Metastasis 1988, 6:115-120. 
10. Roxas M, Jurenka J: Colds and influenza: a review of diagnosis and conventional, botanical, and nutritional considerations. Altern Med Rev 2007, 12:25-48.

11. Classen B, Thude S, Blaschek W, Wack M, Bodinet C: Immunomodulatory effects of arabinogalactan-proteins from Baptisia and Echinacea. Phytomedicine 2006, 13:688-694.

12. Luettig B, Steinmuller C, Gifford GE, Wagner H, Lohmann-Matthes ML: Macrophage activation by the polysaccharide arabinogalactan isolated from plant cell cultures of Echinacea purpurea. J Natl Cancer Inst 1989, 81:669-675.

13. Currier NL, Lejtenyi D, Miller SC: Effect over time of in-vivo administration of the polysaccharide arabinogalactan on immune and hemopoietic cell lineages in murine spleen and bone marrow. Phytomedicine 2003, 10:145-153.

14. D'Adamo P: Larch Arabinogalactan is a Novel Immune Modulator. $J$ Naturopath Med 1996, 4:32-39.

15. Grieshop CM, Flickinger EA, Fahey GC Jr: Oral administration of arabinogalactan affects immune status and fecal microbial populations in dogs. J Nutr 2002, 132:478-482.

16. Kim LS, Waters RF, Burkholder PM: Immunological activity of larch arabinogalactan and Echinacea: a preliminary, randomized, double-blind, placebo-controlled trial. Altern Med Rev 2002, 7:138-149.

17. Nantz M, Painter A, Parker E, McGill C, Percival S: Evaluation of arabinogalactan's effect on human immunity. FASEB J 2001, 15:A633.

18. Causey J, Robinson R, Feirtag J, Fulcher R, Slavin J: Effects of larch arabinogalactan on human peripheral blood mononuclear cells: results from in vivo and in vitro human trials. FASEB J 1999, 13:A589.

19. Thomas C, Moridani M: Interindividual variations in the efficacy and toxicity of vaccines. Toxicology 2009.

20. Chaplin DD: Overview of the immune response. J Allergy Clin Immunol 2010, 125:S3-23.

doi:10.1186/1475-2891-9-32

Cite this article as: Udani et al:: Proprietary arabinogalactan extract increases antibody response to the pneumonia vaccine: a randomized, double-blind, placebo-controlled, pilot study in healthy volunteers. Nutrition Journal 2010 9:32.

\section{Submit your next manuscript to BioMed Central and take full advantage of:}

- Convenient online submission

- Thorough peer review

- No space constraints or color figure charges

- Immediate publication on acceptance

- Inclusion in PubMed, CAS, Scopus and Google Scholar

- Research which is freely available for redistribution 